Mitteilungen der Österreichischen Geographischen Gesellschaft,

157. Jg. (Jahresband), Wien 2015, S. 7-8

\title{
VORWORT DES SCHRIFTLEITERS
}

\author{
Peter JORDAN, Wien*
}

Der Themenschwerpunkt dieses Bandes schöpft aus einer Tagung zum Thema „Zehn Jahre EU-Osterweiterung - geographische Bilanz eines mutigen Schrittes“, die im Dezember 2014 an der Österreichischen Akademie der Wissenschaften stattfand und an der auch die Österreichische Geographische Gesellschaft mitgewirkt hat. Drei der dortigen Vorträge, nämlich solche mehr grundsätzlichen Inhalts und mit einem vergleichenden Blick über die ganze oder größere Teile der Region sind dafür ausgewählt worden: Alessandro VITALE (Mailand [Milano]) diskutiert die Nachbarschaftspolitik der Europäischen Union (EU) und den Bezug der EU zum Prinzip der Territorialität. Piotr Korcelli und seine Tochter Ewa Korcelli-OlejNicZaK (Warschau [Warszawa]) gehen sodann auf die Entwicklung der Metropolen im östlichen Mitteleuropa ein. Stanley BRunN (Lexington, USA) schließt den Themenschwerpunkt mit Antworten auf die Frage ab, was wir denn über politisch relevante geographische Sachverhalte in Bezug auf die Länder und groBen Städte Europas wüssten - was dazu jeweils digital und in Bibliotheken relativ leicht erreichbar wäre. Er kommt aufgrund seiner Untersuchung zu Gliederungen Europas in „Kern“, „Semi-Peripherie“, „Peripherie“ und „Tiefe Peripherie“, die er in konzentrischen Kreisdiagrammen visualisiert.

Die übrigen zwölf Fachartikel des Bandes streuen thematisch über ein weites Feld. Ein erster Block umfasst Themen zu Raumstrukturen und Raumentwicklung. Hier bieten Heinz Fassmann und Stephan Marik-Leвeck (beide Wien) Modellrechnungen zur künftigen Bevölkerungsentwicklung in Österreich und benennen Martin HeINTEL und Norbert Weixlbaumer (beide Wien) die spezifischen Schwierigkeiten grenzüberschreitender Projektarbeit am Beispiel des Naturparks Geschriebenstein-Írottkő an der österreichisch-ungarischen Grenze. Dann demonstrieren Marcel HoRŇÁK (Pressburg [Bratislava]) und Stanislav Kraft (Budweis [České Budějovice]) am Beispiel der Slowakei die gute Verwendbarkeit von PKW-Zählungen zum Ausweis funktionaler Regionen. Schließlich betont Ewa Korcelli-OlejnicZAK (Warschau) die Bedeutung von Umweltqualität als Attraktionsfaktor der Peripherie am Beispiel der Stadtregion von Warschau.

Die nächsten zwei Beiträge widmen sich Themen der Kulturgeographie. Ioana ScRIDON (Augsburg) und Oana-Ramona ILovan (Klausenburg [Cluj-Napoca]) zeigen am Beispiel der kleinen Splittergruppe der Zipser in der rumänischen Marmarosch [Maramureş] wie sich Gruppenidentität gerade auch in Konkurrenz zu anderen örtlichen Gruppen erhalten kann. Irene BERNAL-BARELLA und Nadine SCHARFENoRT (beide Mainz) untersuchen, wie

\footnotetext{
* HR Prof. h.c. Univ.-Doz. Dr. Peter Jordan, Institut für Stadt- und Regionalforschung, Österreichische Akademie der Wissenschaften, Postgasse 7/4/2, A-1010 Wien; E-Mail: peter.jordan@oeaw.ac.at, http://www. oeaw.ac.at/isr
} 
arabische Gäste in Zell am See und Kaprun, zwei Hauptdestinationen arabischer Touristen in Österreich, durch deutsche Touristen wahrgenommen werden.

Den Abschnitt zur Tourismusgeographie im engeren Sinn leitet eine Beschreibung der Tourismusentwicklung in Slowenien durch Anton Gosar (Portorož/Portorose) und Dejan Cigale (Laibach [Ljubljana]) ein. Ivan Šulc und Vuk Tvrtko Opačić (beide Zagreb) legen sodann am Beispiel des kroatischen Süddalmatiens eine Typologie von Tourismusorten vor. Elżbieta GRZELAK-KostULSKA (Thorn [Toruń]) geht mit ihrer Autorengruppe am Beispiel polnischer Kurorte der Frage nach, unter welchen Bedingungen des Transformationsprozesses die Akquisition von EU-Fördermitteln am besten gelungen sei.

In seinem Beitrag zur Fachdidaktik stellt Christian FRIDRICH (Wien) eine Befragung von Wiener Lehrern zur ökonomischen Bildung im österreichischen Geographie- und Wirtschaftskunde-Unterricht vor.

Zwei Beiträge zur Kartographie und Geoinformation schließen den Band ab. Petra SvateK (Wien) untersucht die geopolitische Kartographie im Österreich der Zwischenkriegszeit. Monika RIEGLER stellt mit einem Team der Technischen Universität und der Universität Wien schließlich inhaltliche und methodische Aspekte eines Genderatlasses von Österreich vor.

Unter den Berichten und Kleinen Mitteilungen findet sich der gar nicht so kleine Bericht von Werner BäTzING (Erlangen/Bamberg) über die niederösterreichische Landesausstellung des Jahres 2015, der an dieser Ausstellung als wissenschaftlicher Berater und Experte mitgewirkt hat. Berichte über Tagungen, Personalia und zahlreiche Buchbesprechungen runden den Band wie üblich ab.

Der deutliche Schwerpunkt des Bandes auf dem östlichen Mitteleuropa mit sehr vielen Autoren aus diesen Ländern führt zu einem hohen Anteil englischsprachiger Fachartikel. Damit unterscheidet sich dieser Band deutlich von dem des Vorjahres. Die Tendenz zur englischen Sprache ist damit wohl auch in den „Mitteilungen“ bemerkbar, unterliegt aber je nach thematischem und regionalem Schwerpunkt Schwankungen. Es wird jedenfalls das Bestreben der Schriftleitung sein, der deutschen Sprache weiterhin Gewicht zu geben.

Für das schon sehr große und internationale Herausgeberkomitee der „Mitteilungen“ konnten noch drei weitere Persönlichkeiten gewonnen werden: Axel BorSDORF, ehemals Präsident der ÖGG, bis 2014 Ordinarius für Geographie an der Universität Innsbruck und weiterhin Direktor des Instituts für Interdisziplinäre Gebirgsforschung der Österreichischen Akademie der Wissenschaften; Derek HALL, Professor für Regionalentwicklung in Ayr, Schottland, Verfasser und Herausgeber etlicher tourismusgeographischer Werke über das mittlere und östliche Europa; sowie Helmut WoHLSCHLÄGL, der neugewählte Präsident der ÖGG, der sich in einem Geleitwort vorstellt und sich an Sie wendet.

Karin und Gerhard MAYER waren bei diesem Band erstmals für Satz, Layout und Lektorat zuständig und haben ihr Debüt sehr erfolgreich absolviert. 